\title{
Periodic solutions for second-order difference equations with quadratic-supquadratic condition
}

\author{
Liang Ding ${ }^{1}$, Rongrong $\operatorname{Tian}^{2}$ and Jinlong Wei ${ }^{3 *}$
}

\section{"Correspondence:}

weijinlong@zuel.edu.cn

${ }^{3}$ School of Statistics and

Mathematics, Zhongnan University

of Economics and Law, 430073,

Wuhan, China

Full list of author information is

available at the end of the article

\begin{abstract}
In this paper, we consider the existence of multiple periodic solutions for a class of second-order difference equations with quadratic-supquadratic growth condition at infinity. Moreover, we give three examples to illustrate our main result.
\end{abstract}

MSC: Primary 49J35; secondary 34K13; 34G20

Keywords: Critical points; Linking theorem; Nontrivial periodic solutions; Discrete systems

\section{Introduction and main result}

Discrete equations have been widely employed as mathematical models depicting the nature phenomena in many practical problems including computer sciences, life sciences, mathematical biology, and so on; see [1-5]. Among these discrete equations, discrete nonlinear Schrödinger (DNLS) equations are very important nonlinear lattice models in the nonlinear science, ranging from condensed matter physics to biology [6-11]. Let $\mathbb{N}, \mathbb{Z}$, and $\mathbb{R}$ stand for the sets of all natural numbers, integers, and real numbers, respectively. For $c, d \in \mathbb{Z}$ with $c \leq d$, we define $\mathbb{Z}[c]=\{c, c+1, \ldots\}$ and $\mathbb{Z}[c, d]=\{c, c+1, \ldots, d\}$. It is well known that by the standing wave assumptions DNLS equations can change into the following nonlinear second-order difference equation [12-18]:

$$
\begin{cases}\Delta^{2} x_{n-1}+f\left(n, x_{n}\right)=0, & \forall n \in \mathbb{Z}, \\ f\left(n, x_{n}\right)=\partial_{x_{n}} F\left(n, x_{n}\right), & \forall n \in \mathbb{Z},\end{cases}
$$

where $\Delta x_{n-1}=x_{n}-x_{n-1}, \Delta^{2}=\Delta(\Delta), F \in \mathcal{C}^{1}(\mathbb{R} \times \mathbb{R})$, and $F(n+M, \cdot)=F(n, \cdot)$ for some $M \in \mathbb{N}$

As is known, the critical-point theory is an important tool when dealing with the existence of solutions of differential equations (see [19]), and for discrete system (1.1), there are some results on the existence of periodic solutions in the last few years: especially, for

(c) The Author(s) 2021. This article is licensed under a Creative Commons Attribution 4.0 International License, which permits use, sharing, adaptation, distribution and reproduction in any medium or format, as long as you give appropriate credit to the original author(s) and the source, provide a link to the Creative Commons licence, and indicate if changes were made. The images or other third party material in this article are included in the article's Creative Commons licence, unless indicated otherwise in a credit line to the material. If material is not included in the article's Creative Commons licence and your intended use is not permitted by statutory regulation or exceeds the permitted use, you will need to obtain permission directly from the copyright holder. To view a copy of this licence, visit http://creativecommons.org/licenses/by/4.0/. 
$F(n, X)$ with supquadratic growth condition with respect to $X$ at infinity,

$$
\lim _{|X| \rightarrow+\infty} \frac{F(n, X)}{|X|^{2}}=+\infty
$$

Guo and $\mathrm{Yu}$ [18] developed a new approach to obtain the existence and multiplicity of periodic solutions to discrete system (1.1). Later, for $F(n, X)$ with subquadratic growth condition with respect to $X$ at infinity, Guo and $\mathrm{Yu}$ [20] proved the existence of nontrivial periodic solutions. For the case of $F(n, X)$ with quadratic-supquadratic growth condition in $X$ at infinity,

$$
\liminf _{|X| \rightarrow+\infty} \frac{|F(n, X)|}{|X|^{2}}=\kappa>0,
$$

in 2004, under the assumption that $\kappa$ depends on $M$ (especially, $\kappa(M)>2$ for even $M$ ), Zhou, Yu, and Guo [21] improved the Guo-Yu method of [18] and obtained the existence of two nontrivial $M$-periodic solutions for discrete system (1.1); for more details on the existence of multiple nontrivial $M$-periodic solutions with quadratic-supquadratic condition, we refer to [22, 23]. Moreover, the existence of one nontrivial solution for general nonlinear difference equations, that is, discrete $\phi$-Laplacian equations with quadraticsupquadratic condition, is considered in $[13,15,24]$. For other related works, we refer to $[14,16,17,25-31]$.

Note that in [21], for $F$ with quadratic-supquadratic condition with respect to $X$ at infinity, by introducing the smallest and largest eigenvalues of the matrix

$$
\left(\begin{array}{cccccc}
2 & -1 & 0 & \cdots & 0 & -1 \\
-1 & 2 & -1 & \cdots & 0 & 0 \\
0 & -1 & 2 & \cdots & 0 & 0 \\
\vdots & \vdots & \ddots & \vdots & \vdots & \vdots \\
-1 & 0 & 0 & \cdots & -1 & 2
\end{array}\right)_{M \times M}
$$

that is,

$$
\lambda_{\min }=2\left(1-\cos \frac{2 \pi}{M}\right), \quad \lambda_{\max }= \begin{cases}4 & \text { when } M \text { is even } \\ 2\left(1+\cos \frac{\pi}{M}\right) & \text { when } M \text { is odd }\end{cases}
$$

Zhou, Yu, and Guo obtained the existence of two nontrivial $M$-periodic solutions for system (1.1). Four years later, Xue and Tang obtained the following more general result for $F$ with quadratic-supquadratic condition with respect to $X$ at infinity.

Theorem 1.1 ([23, Theorem 2]) Suppose that $F(n, X)$ satisfies

$\left(F_{1}\right)$ There are constants $\delta>0$ and $k \in\left[0,\left[\frac{M}{2}\right]-1\right] \cap \mathbb{Z}$ such that for any $|X| \leq \delta$ and $t \in \mathbb{Z}[1, M]$,

$$
\frac{1}{2} \mu_{k}|X|^{2} \leq F(n, X) \leq \frac{1}{2} \mu_{k+1}|X|^{2},
$$

where $\mu_{k}=2-2 \cos k w, w=\frac{2 \pi}{M}, M>2$, and [.] denotes the Gauss function; 
$\left(F_{2}\right)$ For $t \in \mathbb{Z}[1, M]$, there exists a constant $\beta \in\left(\frac{\lambda_{\max }}{2},+\infty\right)$ such that

$$
\lim _{|X| \rightarrow+\infty} \inf \frac{F(n, X)}{|X|^{2}} \geq \beta
$$

Then system (1.1) has at least two nontrivial M-periodic solutions.

In condition $\left(F_{2}\right), \lambda_{\max }$ is dependent on $M$; when $M$ is even, $\beta>2$, and when $M \geq 3$ is odd, $\beta \geq 1+\cos \pi / M \geq 3 / 2$. Therefore the constant $\beta$ is at least greater than $3 / 2$. Moreover, in condition $\left(F_{1}\right)$, if $k=0$, then when $M=4,0 \leq F(n, X) \leq 1-\cos (2 \pi / 4)=1$, and when $M>4$, $0 \leq F(n, X) \leq 1-\cos (2 \pi / M)<1$. Clearly, the range of parameter values in $\left(F_{1}\right)$ and $\left(F_{2}\right)$ will play a critical role in discrete model (1.1) when proving the existence of periodic solutions. However, all parameters in Theorem 1.1 are limited. In this paper, we want to establish an existence result for periodic solutions without this limitation. The main result of this paper is the following:

Theorem 1.2 Let $M \geq 4$, and let $F(n, X)$ satisfy the following conditions:

$\left(F_{1}^{\prime}\right)$ There exist constants $\delta>0$ and $0<\alpha<1$ such that

$$
0 \leq F(n, X) \leq \alpha|X|^{2} \quad \text { for } n \in \mathbb{N}, X \in \mathbb{R} \text { and }|X| \leq \delta
$$

$\left(F_{2}^{\prime}\right)$ For $t \in \mathbb{Z}[1, M]$, there exists a constant $\beta \in(1,+\infty)$ such that

$$
\lim _{|X| \rightarrow+\infty} \inf \frac{F(n, X)}{|X|^{2}} \geq \beta
$$

Then system (1.1) has at least two nontrivial M-periodic solutions.

Remark 1.1 (i) Since $M \geq 4$, the parameter $\beta$ in Theorem 1.1 can only take values in ( $1+$ $\cos (\pi / 5),+\infty)$, but it can take any value in $(1,+\infty)$ in Theorem 1.2. Moreover, if $M>4$ and $k=0$, then the parameter $\alpha$ in Theorem 1.1 can only take values in $(0,1-\cos (2 \pi / M))$, but in the present paper, we prove that this parameter can take any value in $(0,1)$. In this sense, we extend the ranges of parameters.

(ii) In Theorem 1.1, if $k \neq 0$, then $F(n, X)=O\left(|X|^{2}\right)$ as $|X| \rightarrow 0$, but in Theorem 1.2, as $|X| \rightarrow 0$, both $F(n, X)=O\left(|X|^{2}\right)$ and $F(n, X)=o\left(|X|^{2}\right)$ are admissible.

Remark 1.2 (i) In 2020, under the assumptions that $M \geq 5$ and $F(n, X) \rightarrow-\infty$ as $|X| \rightarrow$ $+\infty$, by using an extended mountain pass theorem, we obtained the existence of two nontrivial $M$-periodic solutions for quadratic-supquadratic vector field $F(n, X)$ (see [22, Theorem 1.1]) in $X$ at infinity. Different from the method in [22, Theorem 1.1], now by constructing a new functional $J_{1}(x)$, two new orthogonal direct sum decompositions, and Linking theorem [19, Theorem 5.3], under the assumptions that $M \geq 4$ and $F(n, X) \rightarrow+\infty$ as $|X| \rightarrow+\infty$, we also obtain the existence of two nontrivial $M$-periodic solutions for quadratic-supquadratic condition in $X$ at infinity.

(ii) The method improved here may be applied to the general difference equations [13, $15,21,24,28,29$ ], and under general quadratic-supquadratic growth conditions at infinity, we may also obtain the existence of multiple periodic solutions. 
Now we give three examples to explain Theorem 1.2. First, we give an example for completely quadratic condition with respect to $X$ at infinity.

Example 1 Let $F$ be given by

$$
F(n, X)=a_{1}|X|^{2}(\phi(n)+D)
$$

where $a_{1}$ is an arbitrary constant that belongs to $(0,1)$, a constant $D>0$, and $\phi(n)$ is a continuous $M$-periodic function satisfying $|\phi(n)|<D$ for every $n$. Now $F(n, X)$ satisfies all assumptions in Theorem 1.2. Thus (1.1) has at least two nontrivial $M$-periodic solutions.

Second, we give an example for quadratic-supquadratic condition with respect to $X$ at infinity.

Example 2 Let $F$ be given by

$$
F(n, X)=\left(a_{4}|X|^{2}-a_{5}|X|^{4}+a_{6}|X|^{6}\right)(\phi(n)+D),
$$

where $a_{4}, a_{5}, a_{6}$ are arbitrary constants that belong to $(0,1)$, a constant $D>0$, and $\phi(n)$ is as in Example 1. Then $F(n, X)$ satisfies all assumptions in Theorem 1.2. Thus (1.1) has at least two nontrivial $M$-periodic solutions.

Remark 1.3 Since the constants $a_{1}$ and $a_{4}$ in Examples 1 and 2 are arbitrary, these examples cannot be solved by Theorem 1.1.

Finally, we give an example for completely supquadratic condition with respect to $X$ at infinity.

Example 3 Let $F$ be given by

$$
F(n, X)=\left(a_{2}|X|^{4}+a_{3}|X|^{6}\right)(\phi(n)+D),
$$

where $a_{2}$ and $a_{3}$ are arbitrary constants that belong to $(0,1)$, a constant $D>0$, and $\phi(n)$ is as in Example 1. Then $F(n, X)$ satsifies all assumptions in Theorem 1.2. Thus (1.1) has at least two nontrivial $M$-periodic solutions.

\section{Some useful lemmas}

To use the critical point theory to study the existence of periodic solutions to (1.1), we introduce some notions and notations.

- Let $S$ be the set of sequences, that is, $S=\left\{s=\left\{s_{j}\right\}=\left(\ldots, s_{-j}, \ldots, s_{0}, \ldots, s_{j}, \ldots\right), s_{j} \in \mathbb{R}, j \in \mathbb{Z}\right\}$. When $x, y \in S$ and $a, b \in \mathbb{R}, a x+b y$ is given by $a x+b y=\left\{a x_{j}+b y_{j}\right\}_{j=-\infty}^{+\infty}$. For any given positive integer $M, E_{M}$ is a subspace of $S$ defined as

$$
E_{M}=\left\{x=\left\{x_{j}\right\} \in S \mid x_{j+M}=x_{j}, j \in \mathbb{Z}\right\} .
$$


Then, with the common Euclid inner product $\left(\|x\|=\left(\sum_{j=1}^{M}\left(x_{j}\right)^{2}\right)^{\frac{1}{2}}\right), E_{M}$ is an $M$-dimensional Hilbert space. Let

$$
\|x\|_{\alpha}=\left(\sum_{k=1}^{M}\left|x_{k}\right|^{\alpha}\right)^{\frac{1}{\alpha}}, \quad \alpha \in(1, \infty)
$$

Then

$$
\frac{1}{M}\|x\|_{4} \leq\|x\| \leq M\|x\|_{4}, \quad \frac{1}{M}\|x\|_{\frac{3}{2}} \leq\|x\| \leq M\|x\|_{\frac{3}{2}}, \quad \forall x \in E_{M} .
$$

- Let $H$ be a real Hilbert space. $J \in \mathcal{C}^{1}(H)$ is said to satisfy the PS condition if any sequence $\left\{x^{(j)}\right\} \subset H$ for which $\left\{J\left(x^{(j)}\right)\right\}$ is bounded and $J^{\prime}\left(x^{(j)}\right) \rightarrow 0$ as $j \rightarrow \infty$ possesses a convergent subsequence in $H$.

- Different from the known literature used to study the existence of periodic solutions for discrete system (1.1), the result of this paper is not related to the complicated smallest and largest eigenvalues of matrix (1.3), that is, $\lambda_{\min }$ and $\lambda_{\max }$ in (1.4), and now for any $x \in E_{M}$ and $\Delta x=\left(\Delta x_{1}, \Delta x_{2}, \ldots, \Delta x_{M}\right)^{\top} \in \mathbb{R}^{M}$, we let

$$
\sum_{j=1}^{M}\left(\Delta x_{j}\right)^{2}+2 \Delta x_{n-1} \Delta x_{n}=\left(\Delta x_{1}, \Delta x_{2}, \ldots, \Delta x_{M}\right)^{\top} P\left(\Delta x_{1}, \Delta x_{2}, \ldots, \Delta x_{M}\right),
$$

where

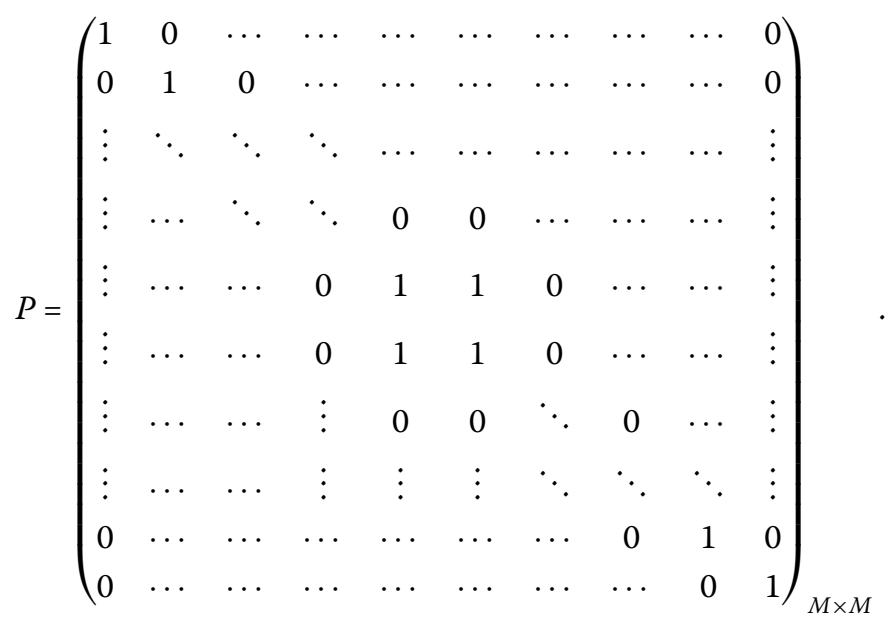

Remark 2.1 Obviously, the matrix $P$ is different from (1.3), and it is easy to get that the eigenvalues of $P$ are $\underbrace{1, \ldots, 1}_{M-2}, 0,2$. Besides, we compute that the matrix $P$ has $M$ linearly independent eigenvectors.

- To obtain the general existence of periodic solutions for second-order difference equation (1.1) with new quadratic-supquadratic condition, we also let $H_{1}=\left\{\left(x_{1}, x_{2}, \ldots, x_{M}\right)^{\top} \in E_{M} \mid \Delta x_{1}=\cdots=\Delta x_{n-2}=\Delta x_{n+1}=\cdots=\Delta x_{M}=0, \Delta x_{n-1}=\right.$ $\left.-\Delta x_{n}=-w \in \mathbb{R}, M \geq 4\right\}$, and $H_{2}=H_{1}^{\perp}$. Then we construct the first new orthogonal direct sum decomposition, $E_{M}=H_{1} \oplus H_{2}$. 
Remark 2.2 Most literatures used the method in $[18,21]$ and $H_{1}$ is trivial, that is, $H_{1}=$ $\{\underbrace{(v, v, \ldots, v)^{\top}}_{M} \mid v \in \mathbb{R}\}$, but in this paper, when $M \geq 4, H_{1}$ is nontrivial, and $\{\underbrace{(v, v, \ldots, v)^{\top}}_{M} \mid$ $v \in \mathbb{R}\} \subseteq H_{1}$, but $\{\underbrace{(v, v, \ldots, v)^{\top}}_{M} \mid v \in \mathbb{R}\} \neq H_{1}$.

Let $M \geq 4$ be a integer, and let $n \in \mathbb{Z}[1, M]$. To prove Theorem 1.2, we define the new functional $J_{1}(x)$, related to the matrix $P$, on $E_{M}$ as follows:

$$
\begin{aligned}
J_{1}(x)= & \frac{1}{2}\|\Delta x\|^{2}-\sum_{j=1}^{M} F\left(j, x_{j}\right)-M^{4}\left[\sum_{j \neq n}\left|x_{j}\right|^{4}+\frac{1}{16}\left|x_{n+1}+x_{n-1}\right|^{4}\right] \\
& +M^{-\frac{3}{2}}\left[\sum_{j \neq n}\left|x_{j}\right|^{\frac{3}{2}}+\frac{1}{2 \sqrt{2}}\left|x_{n+1}+x_{n-1}\right|^{\frac{3}{2}}\right]-\frac{1}{2}(\Delta x)^{\top} P(\Delta x) .
\end{aligned}
$$

Remark 2.3 We have the following identity:

$$
\begin{aligned}
\frac{\partial\left[(\Delta x)^{\top} P(\Delta x)\right]}{\partial x_{n}} & =\frac{\partial\left[\sum_{j \neq n}\left|x_{j}\right|^{4}+\frac{1}{16}\left|x_{n+1}+x_{n-1}\right|^{4}\right]}{\partial x_{n}} \\
& =\frac{\partial\left[\sum_{j \neq n}\left|x_{j}\right|^{\frac{3}{2}}+\frac{1}{2 \sqrt{2}}\left|x_{n+1}+x_{n-1}\right|^{\frac{3}{2}}\right]}{\partial x_{n}}=0 .
\end{aligned}
$$

Clearly, $J_{1} \in \mathcal{C}^{1}\left(E_{M}\right)$. For any $x=\left\{x_{j}\right\}_{j \in \mathbb{Z}} \in E_{M}$, according to Remark 2.3, we compute that

$$
\frac{\partial J_{1}}{\partial x_{n}}=-\left[\Delta^{2} x_{n-1}+f\left(n, x_{n}\right)\right], \quad \forall n \in \mathbb{Z}[1, M]
$$

So, the existence of critical points of $J_{1}$ on $E_{M}$ may imply the existence of periodic solutions of system (1.1).

We now give some useful lemmas, which will serve us later.

Lemma 2.1 Let $\left(F_{2}^{\prime}\right)$ be valid. Then there exists a constant $\gamma^{\prime} \in \mathbb{R}$ such that $F(n, X) \geq$ $\beta|X|^{2}-\gamma^{\prime}$ for $n \in \mathbb{Z}, X \in \mathbb{R}$.

Proof By $\left(F_{2}^{\prime}\right)$, for $\beta>1$, there exist constants $\rho>0$ and $\gamma>0$ such that

$$
F(n, X) \geq \beta|X|^{2}-\gamma \quad \text { for } n \in \mathbb{Z},|X| \geq \rho .
$$

Then letting $\gamma_{1}=\max \left\{\left.|F(n, X)-\beta| X\right|^{2}+\gamma|: n \in \mathbb{Z}| X \mid, \leq \rho\right\}$ and $\gamma^{\prime}=\max \left\{\gamma, \gamma_{1}+\gamma\right\}$, we obtain

$$
F(n, X) \geq \beta|X|^{2}-\gamma^{\prime} \quad \text { for } n \in \mathbb{Z}, X \in \mathbb{R} .
$$

The proof is complete.

Lemma 2.2 Let $\left(F_{2}^{\prime}\right)$ be in force. Then $J_{1}(x)$ is bounded from above on $E_{M}$. 
Proof Let $J_{1}$ be given by (2.1). By Lemma 2.1, for all $x \in E_{M}$, we have

$$
\begin{aligned}
J_{1}(x) \leq & \frac{1}{2}\|\Delta x\|^{2}-\sum_{j=1}^{M}\left(\beta\left(x_{j}\right)^{2}-\gamma^{\prime}\right)-M^{4}\left[\sum_{j \neq n}\left|x_{j}\right|^{4}+\frac{1}{16}\left|x_{n+1}+x_{n-1}\right|^{4}\right] \\
& +M^{-\frac{3}{2}}\left[\sum_{j \neq n}\left|x_{j}\right|^{\frac{3}{2}}+\frac{1}{2 \sqrt{2}}\left|x_{n+1}+x_{n-1}\right|^{\frac{3}{2}}\right]-\frac{1}{2}(\Delta x)^{\top} P(\Delta x) \\
= & \frac{1}{2}\|\Delta x\|^{2}-\beta\|x\|^{2}-M^{4}\left[\sum_{j \neq n}\left|x_{j}\right|^{4}+\frac{1}{16}\left|x_{n+1}+x_{n-1}\right|^{4}\right] \\
& +M^{-\frac{3}{2}}\left[\sum_{j \neq n}\left|x_{j}\right|^{\frac{3}{2}}+\frac{1}{2 \sqrt{2}}\left|x_{n+1}+x_{n-1}\right|^{\frac{3}{2}}\right]+M \gamma^{\prime} \\
& -\frac{1}{2}(\Delta x)^{\top} P(\Delta x) .
\end{aligned}
$$

The eigenvalues of $P$ are $\underbrace{1, \ldots, 1}_{M-2}, 0,2$, and the matrix $P$ has $M$ linearly independent eigenvectors. So we can construct the second new orthogonal direct sum decomposition $\mathbb{R}^{M}=L_{0} \oplus L_{1} \oplus L_{2}$, where

$$
L_{0}=\operatorname{span}\left\{\Delta x \in \mathbb{R}^{M} \mid P \Delta x=0\right\}, \quad L_{1}=\operatorname{span}\left\{\Delta x \in \mathbb{R}^{M} \mid P \Delta x=\Delta x\right\},
$$

and

$$
L_{2}=\operatorname{span}\left\{\Delta x \in \mathbb{R}^{M} \mid P \Delta x=2 \Delta x\right\}
$$

For difference cases, we have the following discussions.

- Case 1: $\Delta x \in L_{0}$. In this case, $(\Delta x)^{\top} P(\Delta x)=0$ and $\Delta x_{n-1}=-\Delta x_{n}$. Then $x_{1}=\ldots=x_{n-1}=$ $x_{n+1}=\ldots=x_{M}$. Thus by $(2.2)$ we have

$$
\begin{aligned}
J_{1}(x) \leq & x_{n}^{2}+x_{n-1}^{2}+2 x_{n-1} x_{n}-\beta\|x\|^{2}-M^{4}(M-1)\left(\left|x_{1}\right|^{4}-\left|x_{1}\right|^{\frac{3}{2}}\right) \\
& +M \gamma^{\prime} .
\end{aligned}
$$

By the Cauchy-Schwarz inequality, for $\beta>1$, from (2.3) we have

$$
\begin{aligned}
J_{1}(x) \leq & x_{n}^{2}+x_{1}^{2}+\frac{2}{\beta-1} x_{1}^{2}+\frac{\beta-1}{2} x_{n}^{2}-\beta\|x\|^{2}-M^{4}(M-1)\left[\left|x_{1}\right|^{4}\right. \\
& \left.-\left|x_{1}\right|^{\frac{3}{2}}\right]+M \gamma^{\prime} \\
\leq & \frac{\beta+1}{\beta-1} x_{1}^{2}-\frac{\beta-1}{2}\|x\|^{2}-M^{4}(M-1)\left[\left|x_{1}\right|^{4}-\left|x_{1}\right|^{\frac{3}{2}}\right]+M \gamma^{\prime} .
\end{aligned}
$$

Since

$$
\lim _{x_{1} \rightarrow \infty}\left[\frac{\beta+1}{\beta-1} x_{1}^{2}-M^{4}(M-1)\left|x_{1}\right|^{4}+M^{4}(M-1)\left|x_{1}\right|^{\frac{3}{2}}\right]=-\infty,
$$

there exists a constant $M_{1}$ such that $J_{1}(x) \leq M_{1}$. So, when the eigenvalue of $P$ is 0 , the functional $J_{1}(x)$ is bounded from above on $E_{M}$. 
- Case 2: $\Delta x \in L_{2}$.

Now $P(\Delta x)=\Delta x$ and $\Delta x_{n-1}=\Delta x_{n}=0$. Therefore $x_{n}=x_{n+1}=x_{n-1}$, and

$$
\begin{aligned}
J_{1}(x) & \leq-\beta\|x\|^{2}-M^{4}\|x\|_{4}^{4}+M^{-\frac{3}{2}}\|x\|_{\frac{3}{2}}^{\frac{3}{2}}+M \gamma^{\prime} \\
& \leq-\beta\|x\|^{2}-\|x\|^{4}+\|x\|^{\frac{3}{2}}+M \gamma^{\prime}
\end{aligned}
$$

So there exists a constant $M_{2}$ such that $J_{1}(x) \leq M_{2}$.

- Case 3: $\Delta x \in L_{3}$.

Now $P(\Delta x)=2 \Delta x$, so $\Delta x_{1}=\ldots=\Delta x_{n-2}=\Delta x_{n+2}=\ldots=\Delta x_{M}=0, \Delta x_{n-1}=\Delta x_{n}$. Thus $2 x_{n}=$ $x_{n+1}+x_{n-1}$, and

$$
J_{1}(x) \leq-\frac{1}{2}\|\Delta x\|^{2}-\beta\|x\|^{2}-\|x\|^{4}+\|x\|^{\frac{3}{2}}+N \gamma^{\prime} \leq M_{2}
$$

Take $M_{3}=\max \left\{M_{1}, M_{2}\right\}$. Then by Cases $1-3$ we get $J_{1}(x) \leq M_{3}$. Now the proof of Lemma 2.2 is complete.

Lemma 2.3 Let hypothesis $\left(F_{2}^{\prime}\right)$ be in force. Then $J_{1}$ satisfies the PS condition.

Proof Let $\left\{J_{1}\left(x^{(j)}\right)\right\}$ be a bounded sequence from bellow, that is, there exists a positive constant $M_{4}$ such that

$$
J_{1}\left(x^{(j)}\right) \geq-M_{4}, \quad \forall j \in N
$$

From (2.4)-(2.6) we have the following inequality:

$$
J_{1}\left(x^{(j)}\right) \leq \begin{cases}\frac{\beta+1}{\beta-1}\left|x_{1}^{(j)}\right|^{2}-\frac{\beta-1}{2}\left\|x^{(j)}\right\|^{2}-M^{4}(M-1)\left[\left|x_{1}^{(j)}\right|^{4}-\left|x_{1}^{(j)}\right|^{\frac{3}{2}}\right] & \\ \quad+M \gamma^{\prime} & \text { when } \Delta x^{(j)} \in L_{0} \\ -\beta\left\|x^{(j)}\right\|^{2}-\left\|x^{(j)}\right\|^{4}+\left\|x^{(j)}\right\|^{\frac{3}{2}}+M \gamma^{\prime} & \text { otherwise, }\end{cases}
$$

which implies

$$
\begin{cases}\frac{\beta-1}{2}\left\|x^{(j)}\right\|^{2}+M^{4}(M-1)\left[\left|x_{1}^{(j)}\right|^{4}-\left|x_{1}^{(j)}\right|^{\frac{3}{2}}\right]-\frac{\beta+1}{\beta-1}\left|x_{1}^{(j)}\right|^{2} & \\ \leq M \gamma^{\prime}+M_{4} & \text { when } \Delta x^{(j)} \in L_{0} \\ \beta\left\|x^{(j)}\right\|^{2}+\left\|x^{(j)}\right\|^{4}-\left\|x^{(j)}\right\|^{\frac{3}{2}} \leq M \gamma^{\prime}+M_{4} & \text { otherwise. }\end{cases}
$$

From (2.7) it is not difficult to deduce that there exists a constant $M_{5}$ such that $\left\|x^{(j)}\right\| \leq$ $M_{5}$, that is, $\left\{x^{(j)}\right\}$ is bounded in $E_{M}$. Since $E_{M}$ is finite-dimensional, there exists a subsequence of $\left\{x^{(j)}\right\}$ (not labeled) convergent in $E_{M}$, so the PS condition is satisfied.

Lemma 2.4 ([19, Theorem 5.3]; Linking theorem) Let $H$ be a real Hilbert space, $H=H_{1} \oplus$ $\mathrm{H}_{2}$, where $\mathrm{H}_{2}$ is a finite-dimensional subspace of $H$. Assume that $J \in \mathcal{C}^{1}(H)$ satisfies the PS condition and

$\left(\mathrm{A}_{1}\right)$ there exist constants $\sigma>0$ and $\rho>0$ such that $\left.J\right|_{\partial B_{\rho} \cap H_{1}} \geq \sigma$;

$\left(\mathrm{A}_{2}\right)$ there are $e \in \partial B_{1} \cap H_{1}$ and a constant $R_{1}>\rho$ such that $\left.J\right|_{\partial Q} \leq 0$, 
where $Q=\left(\bar{B}_{R_{1}} \cap H_{2}\right) \oplus\left\{r e \mid 0<r<R_{1}\right\}, B_{\rho}$ denotes the open ball in $H$ with radius $\rho$ and centered at 0 , and $\partial B_{\rho}$ is its boundary. Then J possesses a critical value $c \geq \sigma$, where

$$
c=\inf _{h \in \Gamma} \max _{u \in Q} J(h(u)), \quad \Gamma=\left\{h \in C(\bar{Q}, H)|h|_{\partial Q}=i d\right\}
$$

and id denotes the identity operator.

\section{Proof of Theorem 1.2}

Based upon Lemmas 2.2-2.4, we divide the proof into four steps.

Step 1. We show that $\left(A_{1}\right)$ in the Linking theorem holds.

When $x \in H_{1}$ and $\Delta x \in L_{0}$, then by $\left(F_{1}^{\prime}\right)$

$$
\begin{aligned}
J_{1}(x) \geq & \frac{1}{2}\|\Delta x\|^{2}-\alpha\|x\|^{2}-M^{4}\left[\sum_{j \neq n}\left|x_{j}\right|^{4}+\frac{1}{16}\left|x_{n+1}+x_{n-1}\right|^{4}\right] \\
& +M^{-\frac{3}{2}}\left[\sum_{j \neq n}\left|x_{j}\right|^{\frac{3}{2}}+\frac{1}{2 \sqrt{2}}\left|x_{n+1}+x_{n-1}\right|^{\frac{3}{2}}\right] \\
= & \left(x_{n}^{2}+x_{n-1}^{2}+2 x_{n-1} x_{n}\right)-\alpha\|x\|^{2}-M^{4}\left[\sum_{j \neq n}\left|x_{j}\right|^{4}+\frac{1}{16} \mid x_{n+1}\right. \\
& \left.+\left.x_{n-1}\right|^{4}\right]+M^{-\frac{3}{2}}\left[\sum_{j \neq n}\left|x_{j}\right|^{\frac{3}{2}}+\frac{1}{2 \sqrt{2}}\left|x_{n+1}+x_{n-1}\right|^{\frac{3}{2}}\right] .
\end{aligned}
$$

Using the Cauchy-Schwarz inequality $2 x_{n-1} x_{n} \leq \frac{1-\alpha}{2} x_{n-1}^{2}+\frac{2}{1-\alpha} x_{n}^{2}$ and $0<\alpha<1$, from (3.1) we arrive at

$$
J_{1}(x) \geq \frac{1-\alpha}{2} x_{n}^{2}-\frac{1+\alpha}{1-\alpha} x_{1}^{2}-(M-1) \alpha x_{1}^{2}-M^{5} x_{1}^{4}+x_{1}^{\frac{3}{2}} / \sqrt{M} .
$$

On the other hand, when $\|x\| \leq \delta,\left(\sum_{j \neq n} x_{j}^{2}\right)^{1 / 2} \leq \delta$, so if we choose $\delta$ sufficiently small, then from (3.2) we conclude

$$
\begin{aligned}
J_{1}(x) & \geq \frac{1-\alpha}{2} x_{n}^{2}+\frac{1}{2 \sqrt{M}} x_{1}^{\frac{3}{2}} \\
& \geq \frac{1-\alpha}{2} x_{n}^{2}+\frac{(1-\alpha)(M-1)}{2} x_{1}^{2}=(1-\alpha)\|x\|^{2} .
\end{aligned}
$$

Taking $\sigma=(1-\alpha) \delta^{2}$, we have

$$
J_{1}(x) \geq \sigma>0, \quad \forall x \in H_{1} \cap \partial B_{\delta} .
$$

Thus condition $\left(A_{1}\right)$ in Lemma 2.4 is satisfied.

Step 2. We show that $\left(A_{2}\right)$ holds.

By Lemma 2.3, $J_{1}(x)$ meets the PS condition. Taking $e \in \partial B_{1} \cap H_{1}$, for any $z \in H_{2}, r \in \mathbb{R}$. Let $x=r e+z$. By $(2.4)-(2.6)$

$$
J_{1}(x) \leq \begin{cases}\frac{\beta+1}{\beta-1}\left|x_{1}\right|^{2}-\frac{\beta-1}{2}\|x\|^{2}-M^{4}(M-1)\left[\left|x_{1}\right|^{4}-\left|x_{1}\right|^{\frac{3}{2}}\right]+M \gamma^{\prime} & \text { when } x \in H_{1}, \\ -\beta\|x\|^{2}-\|x\|^{4}+\|x\|^{\frac{3}{2}}+M \gamma^{\prime} & \text { when } x \in H_{2} .\end{cases}
$$


Observing that as $\|x\| \rightarrow \infty$, the right-hands in (3.5) are approaching to negative infinity, and thus there exists a big enough constant $R_{2}>0$ such that $J_{1}(x) \leq 0$ for all $x \in \partial Q$, where

$$
Q=\left(\bar{B}_{R_{2}} \cap H_{2}\right) \oplus\left\{r e \mid 0<r<R_{2}\right\} .
$$

Step 3. Existence of the first nontrivial $N$-periodic solution.

By Linking theorem (Lemma 2.4), $J_{1}$ has a critical value $c \geq \sigma>0$, where

$$
c=\inf _{h \in \Gamma} \max _{x \in Q} J_{1}(h(x)), \quad \Gamma=\left\{h \in C\left(\bar{Q}, E_{M}\right)|h|_{\partial Q}=i d\right\} .
$$

Step 4. Existence of the second nontrivial $N$-periodic solution.

Inequalities (2.4)-(2.6) imply $\lim _{\|x\| \rightarrow \infty} J_{1}(x)=-\infty$. Let $c_{0}=\sup _{x \in E_{M}} J_{1}(x)$. By the continuity of $J_{1}$ on $E_{M}$ there exists $\bar{x} \in E_{M}$ such that $J_{1}(\bar{x})=c_{0}$ and $\bar{x}$ is a critical point of $J_{1}$. Combining (3.4), we have $J_{1}(\bar{x})=c_{0}>0$. Note that when $x_{1}=\cdots=x_{M}=0$, by (2.1) and condition $\left(F_{1}^{\prime}\right)$ we get $J_{1}(x)=-\sum_{j=1}^{M} F\left(j, x_{j}\right) \leq 0$. So the critical point associated with the critical value $c_{0}$ of $J_{1}$, is a nontrivial $M$-periodic solution of system (1.1).

The rest of the proof of the other nontrivial $M$-periodic solution is similar to that of [18, Theorem 1.1], and we omit it.

By now the proof of Theorem 1.2 is complete, which means that discrete system (1.1) has at least two nontrivial $M$-periodic solutions.

Acknowledgements

The authors are thankful to the editor and anonymous referees for their valuable comments and suggestions.

\section{Funding}

The first author was partially supported by the research funding project of Guizhou Minzu University (GZMU[2019]QN04) and the Guizhou Province Science and Technology Department (ZK[2021]003). The second author was partially supported by the National Natural Science Foundation of China (No. 11901442). The third author was partially supported by National Science Foundation of China (No. 11501577).

Availability of data and materials

Data sharing not applicable to this paper as no datasets were generated or analyzed during the current study.

Competing interests

The authors declare that they have no competing interests.

Authors' contributions

The authors contributed equally to this paper. All authors read and approved the final manuscript.

\section{Author details}

${ }^{1}$ School of Data Science and Information Engineering, Guizhou Minzu University, 550025, Guiyang, China. ${ }^{2}$ College of Science, Wuhan University of Technology, 430070, Wuhan, China. ${ }^{3}$ School of Statistics and Mathematics, Zhongnan University of Economics and Law, 430073, Wuhan, China.

\section{Publisher's Note}

Springer Nature remains neutral with regard to jurisdictional claims in published maps and institutional affiliations.

Received: 9 November 2020 Accepted: 22 August 2021 Published online: 06 September 2021

\section{References}

1. Agarwal, R.P.: Difference Equations and Inequalities: Theory, Methods and Applications. Dekker, New York (1992)

2. Elaydi, S.: An Introduction to Difference Equations. Springer, New York (1999)

3. Yu, J.S., Zheng, B.: Modeling Wolbachia infection in mosquito population via discrete dynamical models. J. Differ. Equ. Appl. 25, 1549-1567 (2019)

4. Shi, Y.T., Yu, J.S.: Wolbachia infection enhancing and decaying domains in mosquito population based on discrete models. J. Biol. Dyn. 14, 679-695 (2020)

5. Long, Y.H., Wang, L.: Global dynamics of a delayed two-patch discrete SIR disease model. Commun. Nonlinear Sci. Numer. Simul. 83, 105117 (2020) 
6. Flach, S., Gorbach, A.V.: Discrete breathers—advances in theory and applications. Phys. Rep. 467, 1-116 (2008)

7. Fleischer, J.W., Carmon, T., Segev, M., Efremidis, N.K., Christodoulides, D.N.: Observation of discrete solitons in optically induced real time waveguide arrays. Phys. Rev. Lett. 90, 023902 (2003)

8. Fleischer, J.W., Segev, M., Efremidis, N.K., Christodoulides, D.N.: Observation of two-dimensional discrete solitons in optically induced nonlinear photonic lattices. Nature 422, 147-150 (2003)

9. Kopidakis, G., Aubry, S., Tsironis, G.P.: Targeted energy transfer through discrete breathers in nonlinear systems. Phys. Rev. Lett. 87, 165501 (2001)

10. Livi, R., Franzosi, R., Oppo, G.L.: Self-localization of Bose-Einstein condensates in optical lattices via boundary dissipation. Phys. Rev. Lett. 97, 060401 (2006)

11. Christodoulides, D.N., Lederer, F., Silberberg, Y.: Discretizing light behaviour in linear and nonlinear waveguide lattices. Nature 424, 817-823 (2003)

12. Erbe, L., Jia, B.G., Zhang, Q.Q.: Homoclinic solutions of discrete nonlinear systems via variational method. J. Appl. Anal. Comput. 9, 271-294 (2019)

13. Lin, G.H., Zhou, Z: Homoclinic solutions in periodic difference equations with mixed nonlinearities. Math. Methods Appl. Sci. 39, 245-260 (2016)

14. Lin, G.H., Zhou, Z., Yu, J.S.: Ground state solutions of discrete asymptotically linear Schrödinger equations with bounded and non-periodic potentials. J. Dyn. Differ. Equ. 32, 527-555 (2020)

15. Lin, G.H., Zhou, Z:: Homoclinic solutions of discrete $\phi$-Laplacian equations with mixed nonlinearities. Commun. Pure Appl. Anal. 17, 1723-1747 (2018)

16. Lin, G.H., Yu, J.S., Zhou, Z.: Homoclinic solutions of discrete nonlinear Schrödinger equations with partially sublinear nonlinearities. Electron. J. Differ. Equ. 2019, 96 (2019)

17. Zhang, Q.Q.: Homoclinic orbits for discrete Hamiltonian systems with local super-quadratic conditions. Commun. Pure Appl. Anal. 18, 425-434 (2019)

18. Guo, Z.M., Yu, J.S.: The existence of periodic and subharmonic solutions for second-order suplinear difference equations. Sci. China Math. 46, 506-515 (2003)

19. Rabinowitz, P.H.: Minimax Methods in Critical Point Theory with Applications to Differential Equations. Am. Math. Soc., Rhode Island (1986)

20. Guo, Z.M., Yu, J.S.: The existence of periodic and subharmonic solutions to subquadratic second-order difference equations. J. Lond. Math. Soc. 68, 419-430 (2003)

21. Zhou, Z., Yu, J.S., Guo, Z.M.: Periodic solutions of higher-dimensional discrete systems. Proc. R. Soc. Edinb. A 134, 1013-1022 (2004)

22. Ding, L., Wei, J.L.: Notes on nontrivial multiple periodic solutions for second-order discrete Hamiltonian system. Bull. Malays. Math. Sci. Soc. 43, 4393-4409 (2020)

23. Xue, Y.F., Tang, C.L.: Multiple periodic solutions for superquadratic second-order discrete Hamiltonian systems. Appl. Math. Comput. 196, 494-500 (2008)

24. Lin, G.H., Zhou, Z.: Homoclinic solutions in non-periodic discrete $\phi$-Laplacian equations with mixed nonlinearities. Appl. Math. Lett. 64, 15-20 (2017)

25. Chen, P., Fang, H.: Existence of periodic and subharmonic solutions for second-order $p$-Laplacian difference equations. Adv. Differ. Equ. 2007, 042530 (2007)

26. Wang, D.B., Xie, H.F., Guan, W.: Existence of periodic solutions for nonautonomous second-order discrete Hamiltonian systems. Adv. Differ. Equ. 2016, 309 (2016)

27. Xue, Y.F., Tang, C.L.: Existence of a periodic solution for subquadratic second-order discrete Hamiltonian system. Nonlinear Anal., Theory Methods Appl. 67, 2072-2080 (2007)

28. Ye, Y.W., Tang, C.L.: Periodic solutions for second-order discrete Hamiltonian system with a change of sign in potential. Appl. Math. Comput. 219, 6548-6555 (2013)

29. Mei, P., Zhou, Z., Lin, G.: Periodic and subharmonic solutions for a 2 nth-order $\phi_{c}$-Laplacian difference equation containing both advances and retardations. Discrete Contin. Dyn. Syst., Ser. S, 12, 2085-2095 (2019)

30. Lin, G.H., Zhou, Z:: Periodic and subharmonic solutions for a $2 n$ th-order difference equation containing both advance and retardation with $\phi$-Laplacian. Adv. Differ. Equ. 2014, 74 (2014)

31. Zhou, Z., Yu, J.S.: Homoclinic solutions in periodic nonlinear difference equations with superlinear nonlinearity. Acta Math. Appl. Sin. Engl. Ser. 29, 1809-1822 (2013)

\section{Submit your manuscript to a SpringerOpen ${ }^{\circ}$ journal and benefit from:}

- Convenient online submission

- Rigorous peer review

- Open access: articles freely available online

- High visibility within the field

- Retaining the copyright to your article

Submit your next manuscript at $\downarrow$ springeropen.com 